\title{
Solar and Stellar Magnetic Fields
}

The IAU-Symposium No. 102 "Solar and Stellar Magnetic Fields: Origins and Coronal Effects" was held during August 2-6, 1982 , at the ETH in Zürich with about 180 participants from 23 countries who met to discuss a variety of aspects of solar and stellar activity. This joint meeting of scientists interested in solar and stellar physics demonstrated the fruitfulness of the "solarstellar connection". Solar physics and the physics of active stars (and nearly all stars are active in some respect) have fertilized each other, both in theory and observation. This was strongly supported by the exciting new developments in X-ray and UV astronomy from space.

The 16 review papers, 38 contributed papers and 61 posters were presented in six main sessions which covered the topics of magnetic fields in stellar atmospheres, rotation-activity-cycle-age connection, theory of stellar magnetic field generation, the role of magnetic fields in the structure and energy balance of stellar atmospheres as well as stellar winds and spindown in late-type stars.

As far as theory is concerned, the topics which found most interest were the structure of photospheric magnetic flux tubes, new developments in dynamo theory, the angular momentum distribution in stellar interiors and especially the electrodynamics of coronal loop heating. For all these topics it became clear that new results (many of them presented at the Symposium for the first time) have improved significantly our understanding.

Many of the observational topics of the meeting were centred around the detailed description and discussion of the stellar $\mathrm{Ca}+\mathrm{H}$ - and $\mathrm{K}$-emission measurements performed since more than a decade at the Mt. Wilson observatory in the USA. From the data obtained by this routine programme, cycles of activity and rotation periods for several late-type main-sequence stars have been found.

Efforts were reported by several groups to determine magnetic field strengths on late-type stars by comparing line profiles of magnetically sensitive and non-sensitive lines. The first results - especially those from infrared observations - are very promising.

Solar magnetograms with space observations of about 0.5 arc $s$ were shown, but the questionable inner network field of 100 $\mathrm{G}$ strength could not be demonstrated clearly. Time analysis of solar magnetograms performed by Russian colleagues resulted in several periods around one year, which were also found in a set of radio radiation data from the USA.

Highly resolved data of solar active regions were obtained using the very large

array of radio antennas. The same equipment also allows stellar radio radiatio, in the $\mathrm{cm}$ wavelength region to be detected.

While an unexpected rotational braking of $\mathrm{G} 5$ giants was found, only an upper limit of the stellar differential rotation in $\mathrm{F}$ type stars was reported, which is much smaller than the value predicted by theoreticians.

Many new details of solar observations were not given in the reviews or contributed papers, but within the large poster exhibition: For instance, the cyclic dependence of the umbra-photosphere intensity ratio is now observed during parts of two cycles and a dependence of meri-

\section{Boundary and Interior Layers}

The second international conference on Boundary and Interior Layers - Computational and Asymptotic Methods, BAIL II, was held in Trinity College, Dublin, Ireland, from 16 to 18 June, 1982, under the auspices of the Numerical Analysis Group. The 82 participants came from 18 countries and included for the first time strong delegations from the USSR and China.

The conference was cosponsored by the American Institute of Aeronautics and Astronautics, the American Meteorological Society, the Institute for Numerical Computation and Analysis and the Irish Mathematical Society.

The aim of this series of conferences is to bring together biologists, chemists, engineers, mathematicians, physicists and other scientists who encounter problems having solutions which exhibit boundary or interior layer behaviour. Both computational and asymptotic methods were discussed extensively at BAIL II, and the degree of difficulty of the problems to which these were applied showed a marked increase over that of the first conference in the series.

The eleven keynote speakers presented papers covering a wide variety of applications and several new computational and asymptotic methods. The areas of application included plasmas, hydrodynamic shocks, transonic airfoils, free surface problems, viscous flows and a variety of phenomena in meteorology. The new methods were concerned with turning point and parabolic problems having a singular perturbation and stiff and other special initial value problems.

In addition there were 44 contributed papers. Many of these were concerned with boundary and interior layer problems arising in biology, chemistry, elasticity, fluid flow, heat transfer, meteorology and petroleum reservoir modelling. Others discussed various computational and asym- dional motions of recurrent sunspots on the phase of the activity cycle was reported.

Within the summary lecture of the symposium, L.E. Cram pointed out, that from an analysis of the sedimented rocks in Australia, it is clear that the periodicity of the solar activity about 700 million years ago was the same as today. In addition, a typical distance of the Maunder minima of about 300 years was found, suggesting that the next minimum of this type may be around the year 2000 .

This Symposium which was very well organized by the staff of the Institute of Astronomy (ETH Zürich), was a milestone in our understanding of stellar activity.

\section{Schüssler and H. Wöhl}

ptotic topics including uniform numerical methods for problems with a singular perturbation, multigrid methods, defect correction techniques, sparse matrices and eigenvalue problems.

When boundary or interior layers are encountered in practical problems it is often found that standard numerical techniques are inaccurate, too expensive or even divergent. This underlines the importance of devising robust numerical algorithms which take account of such layers. In other cases the occurrence of layers may not have been recognised even though they may in fact be present. It is wise therefore to consider their presence as one possible cause for the degradation in performance of an otherwise well-established numerical algorithm. That such is the case in a wide variety of situations is attested to by the many fascinating papers delivered at the first two conferences in this series.

In association with the BAIL II Conference, an introductory short course was held on the same topic as the conference. This consisted of sixteen tutorial lectures on the various conference themes. An exhibition of books and journals was also arranged.

It is proposed to hold the third conference in the series, BAIL III, from 20 to 22 June, 1984

\section{BIBLIOGRAPHY}

1. Miller J.J.H. (ed.) Computational and Asymp totic Methods for Boundary and Interior Layers. Proceedings of the BAIL /I Conference (Boole Press, Dublin) 1982.

2. Miller J.J.H. (ed.) An Introduction to Computational and Asymptotic Methods for Boundary and Interior Layers. Lecture Notes of a Short Course held in association with the BAIL II Conference (Boole Press, Dublin) 1982. 